\title{
CLOSED CONVEX HYPERSURFACES WITH SECOND FUNDAMENTAL FORM OF CONSTANT CURVATURE
}

\author{
ROLF SCHNEIDER
}

\begin{abstract}
It is shown that the Euclidean spheres are the only closed hypersurfaces in Euclidean space on which the second fundamental form defines a (nondegenerate) Riemannian metric of constant curvature.
\end{abstract}

An oriented hypersurface (always assumed to be sufficiently smooth) in $(n+1)$-dimensional Euclidean space $(n \geqq 2)$ will be called closed if it is compact and without boundary, and convex if its second fundamental form II is positive definite. On a convex hypersurface II defines a Riemannian metric, and it is a natural question to ask for the relations between curvature properties of this metric and the geometrical shape of the hypersurface. We shall prove:

THEOREM 1. A closed convex hypersurface on which the second fundamental form is of constant Riemannian curvature has to be a Euclidean sphere.

For a convex hypersurface $S$ under consideration, let $R_{\text {II }}$ denote the scalar curvature of II and let $A_{\mathrm{II}}$ be the total area of $S$ corresponding to the metric II. If $S$ is closed, it is known to be diffeomorphic to a sphere, and if furthermore II is a metric of constant curvature, the Riemannian space $(S$, II) must be globally isometric to a Euclidean sphere of radius $r$, say. In this case we have $R_{\mathrm{II}}=n(n-1) r^{-2}$ and $A_{\mathrm{II}}=\omega_{n} r^{n}$, where $\omega_{n}$ denotes the total area of the $n$-dimensional unit sphere, hence the equality $R_{\mathrm{II}}=$ $n(n-1)\left(\omega_{n} A_{\text {II }}^{-1}\right)^{2 / n}$ holds. Thus Theorem 1 is a consequence of the following stronger theorem, in which II need not be assumed to be of constant curvature.

THEOREM 2. A closed convex hypersurface in Euclidean space which satisfies

$$
R_{\mathrm{II}} \leqq n(n-1)\left(\omega_{n} A_{\mathrm{II}}^{-1}\right)^{2 / n}
$$

has to be a Euclidean sphere.

Received by the editors October 29, 1971.

AMS 1970 subject classifications. Primary 53A05, 53C40; Secondary 53C20.

Key nords and phrases. Second fundamental form, closed convex hypersurface, Riemannian metric of constant curvature, scalar curvature, Euclidean sphere.

(c) American Mathematical Society 1972 
Of course, for $n=2$ this assertion is not really stronger than that of Theorem 1, since the above inequality and the Gauss-Bonnet theorem (applied to the Riemannian space $\left(S\right.$, II)) lead at once to $R_{\mathrm{II}}=$ const.

Proof of Theorem 2. Let $K$ and $H$ denote Gauss-Kronecker curvature and mean curvature of $S$, respectively. Let $\nabla_{\text {II }}$ be the first Beltrami differential operator (square of the gradient) with respect to II. Then the identity

$$
R_{\mathrm{II}}=n(n-1) H+P-(2 K)^{-2} \nabla_{\mathrm{II}} K
$$

holds, where $P$ is some nonnegative function (to be explained later). Deferring the proof of (2) until later, we deduce the inequality

$$
R_{\mathrm{II}} \geqq n(n-1) K^{1 / n}-(2 K)^{-2} \nabla_{\mathrm{II}} K
$$

as a consequence of $P \geqq 0$ and $H^{n} \geqq K$. Now on the closed hypersurface $S$ the function $K$ attains a maximum. In a point where this happens we have $\nabla_{\mathrm{II}} K=0$ and hence $R_{\mathrm{II}} \geqq n(n-1) K^{1 / n}$. Together with the assumption (1) this leads to

$$
K^{1 / 2} \leqq \omega_{n} A_{\mathrm{II}}^{-1}
$$

Since this inequality holds at a point where $K$ attains its maximum, it holds generally on $S$. Now let $d A_{\mathrm{I}}, d A_{\text {II }}$ denote the area elements on $S$ with respect to the first fundamental form I and the second fundamental form II, respectively. Then we have $K^{1 / 2} d A_{\mathrm{I}}=d A_{\mathrm{II}}$, since $K$ equals the quotient of the determinants of II and I. Integrating over all of $S$, we deduce, from (3),

$$
\int K d A_{\mathrm{I}} \leqq \int \omega_{n} A_{\mathrm{II}}^{-1} K^{1 / 2} d A_{\mathrm{I}}=\omega_{n} A_{\mathrm{II}}^{-1} \int d A_{\mathrm{II}}=\omega_{n} .
$$

Here the left hand integral is also equal to $\omega_{n}$, since it represents the total area of the spherical image of the closed convex surface $S$. We deduce that the equality sign must hold throughout in (3), hence that the function $K$ is constant. But it is well known that the only closed hypersurfaces with constant $K$ are the Euclidean spheres.

It remains to prove equation (2). Here $P$ has the following meaning: Let $T_{i j}^{k}$ denote the difference tensor of the Levi-Civita connections with respect to I and II. In the following we shall use the tensor $b_{i j}$ of the second fundamental form as the fundamental tensor for "raising and lowering the indices" in the sense of classical tensor notation. Then $P$ is defined by $P=T_{i j k} T^{i j k}$, from which its nonnegativity is obvious. If $\nabla^{\text {II }}$ denotes covariant differentiation with respect to II, a direct computation gives (Eisenhart [2, p. 33])

$$
\nabla_{l}^{\mathrm{II}} T_{i j}^{k}-\nabla_{j}^{\mathrm{II}} T_{i l}^{k}=\bar{R}_{i j l}^{k}-R_{i j l}^{k}+T_{i l}^{m} T_{m j}^{k}-T_{i j}^{m} T_{i n l}^{k},
$$


where $\bar{R}_{i j l}^{k}, R_{i j l}^{k}$ are the curvature tensors of II and I, respectively. Contracting once and transvecting with $b^{i l}$, we arrive at

$$
R_{\mathrm{II}}=b^{i l} R_{i l}+\nabla_{i}^{\mathrm{II}} T_{j}^{i j}-\nabla_{j}^{\mathrm{II}} T_{i}^{i j}-T_{i}^{i m} T_{m j}^{j}+T_{i j}^{m_{i}} T_{m}^{i j}
$$

where $R_{i l}$ is the Ricci tensor of I. Let $\Gamma^{\mathrm{I}}$ denote covariant differentiation with respect to $I$. Expressing $\nabla_{k}^{\mathrm{I}} b_{i j}$ and $\Gamma_{k}^{\mathrm{II}} b_{i j}(=0)$ by means of Christoffel symbols we get

$$
T_{i k j}+T_{j k i}=-\nabla_{k}^{\mathrm{I}} b_{i j}
$$

By the Codazzi equations the right-hand side is symmetric in all three indices, hence $T_{i j k}$ (which is symmetric in the first two indices) is symmetric. Therefore equality (4) simplifies to

$$
R_{1 \mathrm{I}}=b^{i l} R_{i l}+P-(2 K)^{-2} \nabla_{\mathrm{II}} K,
$$

where the formula

$$
2 T_{m j}^{j}=\nabla_{m} \log \operatorname{det} \mathrm{I}-\nabla_{m} \log \operatorname{det} \mathrm{II}=-\nabla_{m} \log K
$$

has been used. Finally, the identity $b^{i l} R_{i l}=n(n-1) H$ is a consequence of the Gauss equations

$$
R_{i j l}^{k}=\hat{g}^{k m}\left(b_{m j} b_{i l}-b_{m l} b_{i j}\right)
$$

where $\hat{g}^{k m}$ is the inverse tensor of $\mathbf{I}$.

REMARK 1. The identity (2) could also be deduced from similar computations by Gardner [4, formula (21)], or from a more general equation in [5, formula (2.23)]; for $n=2$ one should also compare E. Cartan [1, p. 11] and Erard [3, p. 7]. For the reader's convenience we gave the short proof in full.

REMARK 2. If on a (not necessarily closed) convex hypersurface we have $R_{1 \mathrm{I}}=1$ and $K=1$ identically, then equation (2) leads to $P=0$ and hence to $\Gamma_{k}^{1} b_{i j}=0$. It follows (Simon-Weinstein [6, Corollary 2.1], that the hypersurface lies on a sphere. This remark generalizes a result of Erard [3, p. 31], who treated the two-dimensional case.

REMARK 3. Theorem 1 is, at least for $n=2$, really a global theorem: There exist convex surfaces in three-dimensional space which are IIisometric to pieces of spheres, but are themselves not pieces of spheres. Such examples have been discovered by Erard [3].

REMARK 4. K. V'OSS [8] has proved that the sphere in three-dimensional space is infinitesimally II-rigid, i.e. every infinitesimal deformation of the 2-sphere which leaves its second fundamental form stationary has to be an infinitesimal motion. 
REMARK 5. It would be interesting to know whether there exists, for the second fundamental form, a rigidity theorem analogous to CohnVossen's theorem on isometric ovaloids: Are two closed convex hypersurfaces congruent if their second fundamental forms coincide under some diffeomorphism? Theorem 1 gives an affirmative answer for the case where one of the hypersurfaces is a sphere. For $n=2$ closed convex surfaces are known to be congruent if not only the second fundamental forms are the same, but also certain functions of the principal curvatures coincide (e.g., their product-which is a well-known theorem of Grove), see Walden [9]. Infinitesimal analogues have been proved by Simon [7].

REMARK 6. The form $K^{-1 /(n+2)}$ II has the remarkable property of being invariant under unimodular affine transformations, therefore it serves as one of the fundamental forms in affine differential geometry. It has been shown that ellipsoids are the only closed convex hypersurfaces on which this form is of constant curvature [5, Satz 4.6].

\section{REFERENCES}

1. E. Cartan, Les surfaces qui admettent une seconde forme fondamentale donnée, Bull. Sci. Math (2) 67 (1943), 8-32. MR 7, 30.

2. L. P. Eisenhart, Riemannian geometry, 4th ed., Princeton Univ. Press, Princeton, N.J., 1960.

3. P. J. Erard, Über die zweite Fundamentalform von Fläch'n im Raum, Doctoral Thesis, ETH, Zürich, 1968.

4. R. B. Gardner, Subscalar pairs of metrics with applications to rigidity and uniqueness of hypersurfaces with a non-degenerate second fundamental form (to appear).

5. R. Schneider, Zur affinen Differentialgeometrie im Grossen. I, Math. Z. 101 (1967), 375-406. MR 36 \#3255.

6. U. Simon and A. Weinstein, Anwendungen der de Rhamschen Zerlegung auf Probleme der lokalen Flächentheoric, Manuscripta Math. 1 (1969), 139-146. MR 39 \#7538.

7. U. Simon, II-Verbic'gungen von Eiflächen, Arch. Math. 22 (1971), 319-324.

8. K. Voss, Isometrie von Flächen bezïglich der zweit'n Fundamentalform, Nachr. Österr. Math. Ges. 91 (1970), 73.

9. R. Walden, Eindeutigkeitssätze für II-isometrische Eiflächen, Math. Z. 120 (1971), 143-147.

Depariment of Mathematics, Technische Universitä? Berlin, Berlin, Germany 